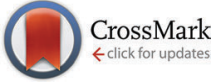

Cite this: J. Mater. Chem. C, 2016, 4, 8778

Received 5th August 2016 Accepted 30th August 2016

DOI: $10.1039 / c 6 t c 03378 d$

www.rsc.org/MaterialsC

\section{Co-flow microfluidic synthesis of liquid crystalline actuating Janus particles $\dagger$}

\author{
T. Hessberger, ${ }^{a}$ L. B. Braun, ${ }^{a}$ F. Henrich, ${ }^{b}$ C. Müller, ${ }^{c}$ F. Gießelmann, ${ }^{c}$ C. Serra ${ }^{d}$ and \\ R. Zentel ${ }^{* a}$
}

In this article the microfluidic synthesis and characterization of micrometer sized actuating Janus particles containing a liquid crystalline elastomer (LCE) is presented. On one side these Janus particles consist of a hydrophobic liquid crystalline part, featuring strong shape changes during the thermotropic phase transition, whereas the other side contains a hydrophilic polyacrylamide network. The synthesis is based upon the dispersion of two immiscible monomer mixtures in a continuously flowing silicone oil, using two glass capillaries side by side to form Janus microdroplets of different morphologies. Furthermore, the systematic adjustment of the morphology of the Janus particles as well as the optimization of the actuation properties is conducted by precise control and variation of the microfluidic parameters. The actuation properties of the particles are studied by polarized optical microscopy (POM), in which relative length changes up to $52 \%$ are investigated for the elongation of LCEs during the phase transition in rodlike Janus particles. Further wide-angle X-ray scattering (WAXS) measurements verify the mesogen's orientation in a bipolar director field, which corresponds to the observed geometry of the Janus particle's shape changes.

\section{Introduction}

The unique properties of Janus particles have attracted the attention of many researchers during the last two decades to derive advantages from the anisotropic and non-centrosymmetric architecture of these particles, which were named after the twinheaded Roman god Janus. The great importance and the manifold possibilities of Janus particles were already demonstrated by de Gennes in his Nobel lecture in $1991 .{ }^{1}$ Since then, a large variety of different synthetic methods have been developed, as well as multiple applications in the fields of materials and life science. ${ }^{2-6}$ Thereby, self-assembly, emulsion techniques, symmetry breaking and phase separation processes enable synthetic strategies for nanometer scaled Janus particles, whereas microfluidic devices, ${ }^{7-10}$ biphasic electrified jetting ${ }^{11}$ or seeded dispersion polymerization $^{12}$ are suitable and well-established methods for the production of various morphologies of polymeric,

\footnotetext{
a Johannes Gutenberg-Universität Mainz, Duesbergweg 10-14, 55099 Mainz, Germany.E-mail: zentel@uni-mainz.de

${ }^{b}$ Max Planck Institute for Polymer Research, Ackermannweg 10, 55128 Mainz, Germany

${ }^{c}$ University of Stuttgart, Pfaffenwaldring 55, 70569 Stuttgart, Germany

${ }^{d}$ Institut Charles Sadron, 23 rue du Loess, BP 84047, 67034 Strasbourg Cedex 2, France

$\dagger$ Electronic supplementary information (ESI) available: Two films demonstrating the preparation of Janus-particles and their actuation are included. See DOI: 10.1039/c6tc03378d
}

micrometer sized Janus particles. The asymmetric architecture permits the combination of two materials in a single micro- or nanometer sized object, each providing different chemical and physical properties, which offers a wide range of unique functionalities. Amphiphilic Janus particles have been studied intensely for their application as solid surfactants and interface stabilizers, catalysts and super-hydrophobic textiles and coatings. ${ }^{13-17}$ Electrical and magneto-responsive Janus particles, featuring asymmetric optical properties, offer their usage for switchable display devices and electronic paper. ${ }^{18-20}$ An increasing interest has grown in stimuli responsive Janus particles, which are sensitive to changes in their physical or chemical environment, such as the temperature, light or pH-value. ${ }^{21-23}$ These Janus particles are able to undergo self-assembly processes, reversible shape changes or even stimuli-driven active propulsion, induced by thermophoresis or catalytic decomposition of a reactive fluid at one hemisphere of these so-called Janus motors. ${ }^{24-27}$ In this work we present a liquid crystalline elastomer (LCE) as the stimuli responsive, actuating part of Janus particles.

LCEs are slightly crosslinked liquid crystalline polymer networks, which are capable of undergoing reversible and stimuli responsive shape transformations during their phase transition. However, LCEs have never been used as stimuli responsive materials in active Janus particles. Since de Gennes predicted the promising shape changing properties ${ }^{28}$ of LCEs in 1975, they have been focused and reviewed by many 
researchers to this day. ${ }^{29-33}$ Based on the responsivity of LCEs due to temperature changes, light irradiation or solvent swelling, these materials have been identified to be suitable for application as actuators, sensors and microelectromechanical systems (MEMSs). ${ }^{34-39}$ A co-flow microfluidic preparation of actuating particles containing a liquid crystalline elastomer represents an effective method for the synthesis of thermal shape changing, microscaled objects. ${ }^{40-43}$ As co-flow microfluidic setups have already been used for the production of amphiphilic Janus microparticles, ${ }^{44,45}$ the combination of hydrophobic LCEs with hydrophilic polymer networks in a microfluidic device offers a promising method for the synthesis of strongly actuating Janus particles. In this work we present the first microfluidic synthesis of actuating Janus particles containing a liquid crystalline elastomer and describe the control of the morphology as well as the actuation properties of the particles.

\section{Experimental}

\section{Materials and reagents}

The liquid crystalline monomer was synthesized as described in the literature. ${ }^{46}$ The UV-photoinitiators diphenyl(2,4,6-trimethylbenzoyl)phosphine oxide and 2-hydroxy-2-methylpropiophenone, the crosslinking agent $N, N^{\prime}$-methylenebis(acrylamide), the monomer acrylamide, the surfactant sodium dodecylbenzenesulfonate (SDBS) and the silicone oils (100 and $1000 \mathrm{cSt}$ ) were purchased from Aldrich and used as received. The PDMS-PEG (polydimethylsiloxanepolyethylene glycol) copolymer was purchased from ABCR. The crosslinking agent 1,6-hexanediol diacrylate was purchased from Aldrich and distilled before use. The high molecular weight polyacrylamide was synthesized in a free radical polymerization using a standard procedure. $^{47}$

The liquid crystalline monomer mixture LC1 was prepared for the microfluidic synthesis by dissolving the LC-monomer, the crosslinking agent 2-hydroxy-2-methylpropiophenone (10 mol\%) and the photoinitiator diphenyl(2,4,6-trimethylbenzoyl)phosphine oxide ( $2 \mathrm{wt} \%)$ in dichloromethane. The solution was stirred for a short time and the solvent was evaporated. The resulting mixture was melted at $90{ }^{\circ} \mathrm{C}$ and filled into a PTFE tube (ID: $1.59 \mathrm{~mm}$ ), which was plugged into the microfluidic setup inside the water bath. A silicone oil (100 cSt) was used as a hydraulic fluid in a syringe to pump the LC1 mixture into the microreactor.

The aqueous monomer mixtures $\mathbf{A 1}$ and $\mathbf{A} 2$ were prepared by dissolving the monomer acrylamide (40 wt\%), the crosslinking agent $N, N^{\prime}$-methylenebis(acrylamide) (10 mol\%), the photoinitiator 2-hydroxy-2-methylpropiophenone (2 wt\%) and small traces of a red ink in distilled water. For the $\mathbf{A} 2$ mixture 0.5 wt $\%$ of polyacrylamide were added to the solution and stirred for 24 hours. The monomer mixture was filled into a $1 \mathrm{~mL}$ syringe, which was plugged directly into the microreactor via a PTFE tube (ID: $170 \mu \mathrm{m}$ ). For the measurements of the interfacial tension different amounts (from $0.01 \mathrm{wt} \%$ to $1 \mathrm{wt} \%$ ) of the surfactant sodium dodecylbenzenesulfonate or the PDMS-PEG copolymer were added to the solution.

\section{Microfluidic setup}

The setup of the microreactor is illustrated in Fig. 1. Two PEEK T-junctions were connected to each other by a PEEK tube (ID: $340 \mu \mathrm{m}$ ) and tempered to $80{ }^{\circ} \mathrm{C}$ in a water bath. Two glass capillaries (ID: $100 \mu \mathrm{m}$, OD: $165 \mu \mathrm{m}$ ) were situated inside the T-junctions, one to provide the monomer mixture LC1 from the inside of the T-junction (ID: $0.5 \mathrm{~mm}$ ) on the left, and the other one directly connected to a PTFE tube (ID: $170 \mu \mathrm{m}$ ) to provide the monomer mixtures $\mathbf{A 1}$ (or A2). The second T-junction (ID: $1.25 \mathrm{~mm}$ ) on the right was connected to a silicone oil (1000 cSt) filled syringe by a PTFE tube (ID: $500 \mu \mathrm{m}$ ) to provide the continuous phase. Three syringe pumps (Harvard Apparatus "Pump 33") were used for the continuous injection of the monomer mixtures (flow rates: 0.01 to $0.2 \mathrm{~mL} \mathrm{~h}^{-1}$ ) and the silicone oil (flow rates: 0.5 to $3 \mathrm{~mL} \mathrm{~h}^{-1}$ ). The tips of glass capillaries were placed in the PTFE polymerization tube (ID: $500 \mu \mathrm{m}$ or $750 \mu \mathrm{m})$, which led through the water bath and thereafter passed over a hot plate $\left(65^{\circ} \mathrm{C}\right)$. The polymerization tube was irradiated over a distance of $1 \mathrm{~cm}$ on the hot plate using an Oriel LSH302 (500 W) lamp equipped with a band filter (323-385 $\mathrm{nm})$ and a waveguide.

\section{Analysis}

The length measurements of the LCE were carried out via polarized optical microscopy (Olympus BX51) at $130{ }^{\circ} \mathrm{C}$ (isotropic phase: $\mathrm{L}_{\text {Iso }}$ ) and at $80{ }^{\circ} \mathrm{C}$ (amorphous state: $\mathrm{L}_{\mathrm{Am}}$ ). The heating of

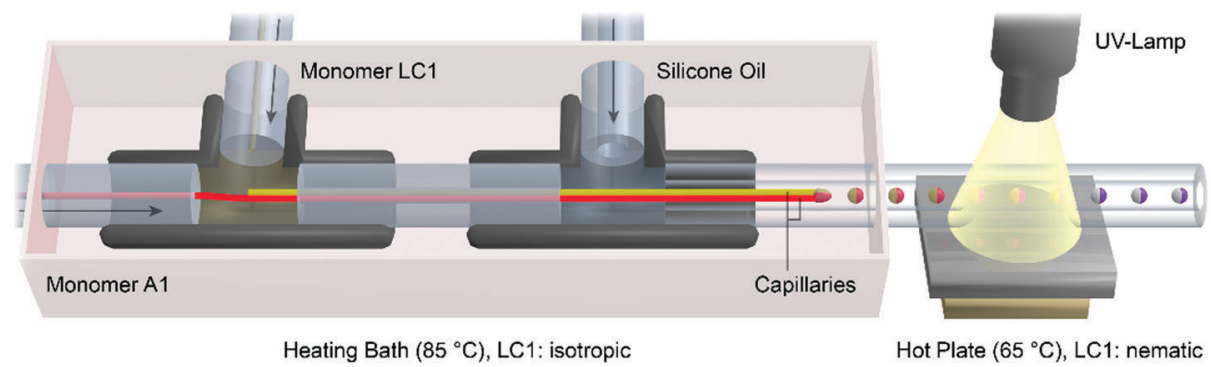

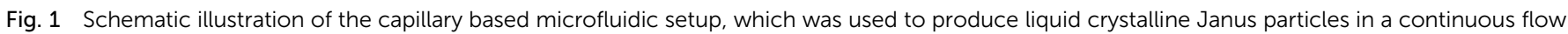

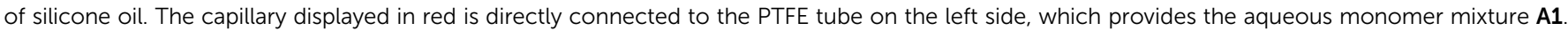

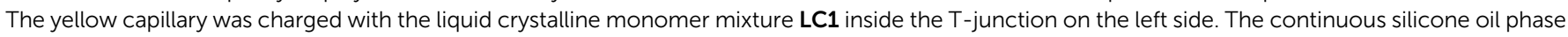
$\mathbf{C P}$ is injected into the $\mathrm{T}$-junction on the right side perpendicular to the glass capillaries. 
Table 1 Measured LCE lengths of the synthesized particles discussed in Sections 2.3 and 3.1. The relative length change is defined as the ratio between the main axis of the LCE in the isotropic state $\left(L_{\mid s o}\right)$ and the amorphous state $\left(L_{A m}\right) . V_{L C}$ is the calculated $L C E$ volume derived from the 3D simulations. The volume ratio between the LC1 part and the $\mathbf{A} \mathbf{1}$ (or $\mathbf{A} \mathbf{2}$ ) part $V_{\mathrm{LC}}: V_{\mathrm{A}}$ was evaluated from the adjusted flow rates of the dispersed phases during the microfluidic synthesis. The area of the boundary surface between the dispersed phases $\mathbf{B}_{\mathbf{L C} / \mathbf{A}}$ was calculated from the particle radius at the boundary surface

\begin{tabular}{lllllll}
\hline & $\begin{array}{l}\mathrm{L}_{\text {Iso }} \\
{[\mu \mathrm{m}]}\end{array}$ & $\begin{array}{l}\mathrm{L}_{\mathrm{Am}} \\
{[\mu \mathrm{m}]}\end{array}$ & $\begin{array}{l}\text { Relative } \\
\text { length change }\end{array}$ & $\begin{array}{l}V_{\mathrm{LC}} \\
{\left[\mathrm{mm}^{3}\right]}\end{array}$ & $\begin{array}{l}V_{\mathrm{LC}}: V_{\mathrm{A}} \\
\text { Particle no. }\end{array}$ & $\begin{array}{l}\mathbf{B}_{\mathrm{LC} / \mathbf{A}} \\
{\left[\mathrm{mm}^{2}\right]}\end{array}$ \\
\hline 5.b/6.d & 536.6 & 846.2 & 1.58 & 35 & $1: 0$ & - \\
5.c & 223.2 & 271.6 & 1.22 & 14 & $1: 1$ & 0.053 \\
5.d & 321.3 & 427.2 & 1.33 & 22 & $4: 1$ & 0.064 \\
6.a & 242.3 & 293.8 & 1.21 & 18 & $2: 1$ & 0.047 \\
6.b & 365.1 & 261.1 & 1.40 & 18 & $3: 1$ & 0.054 \\
6.c & 846.2 & 536.6 & 1.52 & 40 & $3: 1$ & 0.062
\end{tabular}

the particles was performed on a microscope hot-stage (Linkam TMS 94). Particle images were taken using a microscope camera (Olympus ColorView II) and analyzed using microscope imaging software (Olympus cell $\wedge^{\wedge} \mathrm{D}$ ). Table 1 illustrates the measured data for the LCE lengths and the calculated relative length changes. The actual LCE volume $V_{\mathrm{LC}}$ was determined by simulating the body of rotation from the particle's cross-sectional area, which was observed from the optical images of the particles. The simulation of the body of rotation and the calculation of $V_{\mathrm{LC}}$ were carried out using the open source 3D rendering program "Blender 2.76".

The measurements of the interfacial tension between the $\mathbf{A 1}$ phases and the silicone oil (1000 cSt) were carried out using a tensiometer DCAT 11 (DATAphysics, Germany) equipped with a Wilhelmy plate made of platinum-iridium (PT11: length $10 \mathrm{~mm}$, width $19.9 \mathrm{~mm}$, and thickness $0.2 \mathrm{~mm}$ ) at $85^{\circ} \mathrm{C}$. Every measurement started with an automatic weighing of the Wilhelmy plate in the A1 phase. Thereafter the silicone oil was added and the interfacial tension between the two phases was measured. Every surfactant concentration in A1 was measured separately.

The WAXS studies were performed on a Janus particle, which was synthesized under the same microfluidic conditions as particle 6.a. The measurements were carried out using a "Nanostar-System" from the company Bruker AXS equipped with two crossed Goebel mirrors, a Kristalloflex 770 generator with a copper anode and a HiStar flat panel detector with a resolution of $1024 \times 1024$ pixels and a diameter of $11.5 \mathrm{~cm}$. The diameter of the point-shaped X-ray beam is defined by an aperture plate of $100 \mu \mathrm{m}$.

\section{Results and discussion}

\section{Microfluidic preparation of Janus particles}

Janus particles should be fabricated in a co-flow microfluidic reactor. The principle of the formation of Janus droplets is based on the dispersion of two monomer mixtures in a continuously flowing silicone oil phase (CP). The prerequisite for

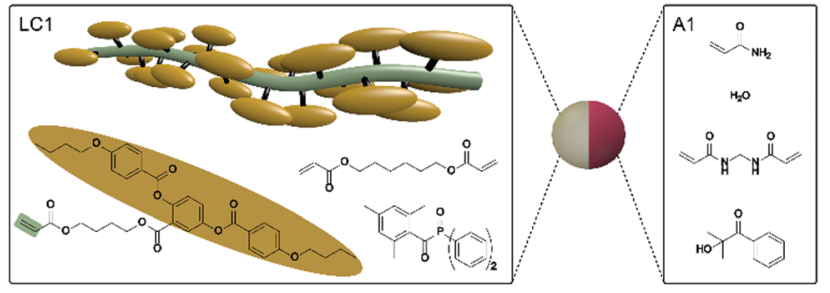

Fig. 2 Segment of a side-on liquid crystalline polymer chain and compounds of the liquid crystalline monomer mixture LC1 (left) and the aqueous monomer mixture $\mathbf{A} \mathbf{1}$ (right).

biphasic Janus droplet formation is the immiscibility of the dispersed monomer phases with each other, in addition to the immiscibility with the continuous silicone oil phase. For this reason a hydrophobic liquid crystalline monomer mixture (LC1) was chosen to form the actuating LCE part of the particle, whereas an aqueous acrylamide monomer mixture (A1) was selected for the hydrophilic part. Fig. 2 illustrates the components for both parts of the Janus particles. LC1 consists of the polymerizable nematic side-on mesogen (4'-acryloyloxybutyl) 2,5-di(4'-butyloxybenzoyloxy)benzoate, the bifunctional crosslinking agent hexanediol diacrylate $(10 \mathrm{~mol} \%)$ and the photoinitiator lucirin TPO (3 wt\%). This LC-monomer mixture shows a monotropic nematic phase with a clearing temperature of $79-80^{\circ} \mathrm{C}$. It has already been used for the fabrication of several functional materials including actuating microparticles. ${ }^{38,40,46}$ The A1 monomer mixture is composed of a $40 \mathrm{wt} \%$ aqueous solution of acrylamide, the bifunctional crosslinker bisacrylamide ( $5 \mathrm{~mol} \%$ ), the photoinitiator 2-hydroxy-2-methylpropiophenone (3 wt\%) and small traces of an aqueous red ink. Both monomer mixtures are immiscible with each other as well as with the silicone oil of $\mathbf{C P}$ $\left(\eta_{\mathrm{CP}}=9.3 \times 10^{-1} \mathrm{~Pa} \mathrm{~s}\right)$. The dynamic viscosities of both monomer mixtures were measured at $85{ }^{\circ} \mathrm{C}$ and a shear rate of $100 \mathrm{~s}^{-1}$ to be $\eta_{\mathbf{L C 1}}=5.3 \times 10^{-1} \mathrm{~Pa}$ s and $\eta_{\mathbf{A 1}}=4.9 \times 10^{-4} \mathrm{~Pa} \mathrm{~s}$.

The formation of the Janus droplets took place at the tips of two glass capillaries, each providing one of the monomer mixtures (see Video S1, ESI $\dagger$ ). Fig. 1 shows the droplet formation and polymerization process as well as the microreactor's setup, which had already been described for the synthesis of polymeric particles before. ${ }^{10,44,45,48}$ The flow rates of both the monomer mixtures LC1 and A1 and the silicone oil $\mathbf{C P}$, were controlled by syringe pumps connected to the T-junctions via PTFE microtubes. The flow rates of the monomer mixtures were adjusted to magnitudes between 0.01 and $0.20 \mathrm{~mL} \mathrm{~h}^{-1}$, whereas the flow rate of the continuous phase was varied in a range of 0.5 and $3.0 \mathrm{~mL} \mathrm{~h}^{-1}$. The morphology and geometry (volume, diameter and shape) of the Janus droplets were controlled by the ratio of the flow rate of silicone oil to the flow rates of the monomer mixtures with respect to the inner diameter of the polymerization tube. To ensure the necessary fluidity of the liquid crystalline monomer mixture, which is in the solid state at room temperature, the charging of the capillaries inside the T-junctions was carried out in a heating bath at $85{ }^{\circ} \mathrm{C}$. A continuous flow of the viscous silicone oil surrounded the capillaries and caused the tearing of Janus droplets due to the shear rate acting on the 
monomer dispersed phases LC1 and A1. Transportation of the Janus droplets by the silicone oil over a hot plate at $65{ }^{\circ} \mathrm{C}$ cooled down the liquid crystalline monomer mixture from the isotropic to the nematic state. Polymerization and crosslinking of the Janus droplet's monomer mixtures were initiated by UV-irradiation on the hot plate. The resulting crosslinked Janus particles were collected at the end of the polymerization tube, removed from the silicone oil and cleaned in petroleum ether for further analysis.

\section{Control of the geometry and the shape of Janus particles}

2.1 Acorn-like Janus particles. The first synthesis of Janus particles using the co-flow microreactor and the materials described above led to acorn-like shaped, eccentric Janus particles. This kind of particle morphology has been discovered in microfluidic syntheses before, in which different monomer mixtures were used. ${ }^{44,49}$ Fig. 3 illustrates the droplet formation and temporal development of its geometry in the continuous silicone oil flow, as well as a crosslinked Janus particle before and after the thermal phase transition of the LCE. The eccentric Janus droplets consisted of an aqueous core containing monomer mixture A1, surrounded by a shell consisting of mixture LC1. This droplet morphology is formed due to the high interfacial tension between silicone oil and the aqueous monomer mixture A1. Consequently the aqueous phase tries to "hide" inside the liquid crystalline phase. This core-shell structure is deformed by the shear flow in the polymerization tube. ${ }^{50,51}$ After the polymerization, the $\mathbf{A 1}$ core forms an oblate shaped part at the front side of the crosslinked acorn-like particle (Fig. 3), while the LC1 part with the higher viscosity forms a hemispherical shell at the back side of the particle with respect to the flow direction of the continuous phase.

The phase transition of the LCE part is monitored via POM by crossing the diffuse clearing temperature range between $90{ }^{\circ} \mathrm{C}$ and $130{ }^{\circ} \mathrm{C}$. Thereby the phase boundary inside the Janus particle is uncovered as the LCE part got transparent in the isotropic phase. Thus the influence of the phase transition on the shape of the Janus particle can be studied. However it turned out that the shape changes of the LCE-part during the phase transition in these eccentric Janus particles are negligible (relative length changes $<5 \%$ ) compared to actuating LCE particles composed of the same material (see Fig. 3(c)). ${ }^{40,41}$ This small shape variation may be due to two reasons: (1) the director field of the LCE and (2) the movability of the LCE during the phase transition.

On one side the small actuation may be the result of a poor macroscopic director orientation in the LCE-part of the

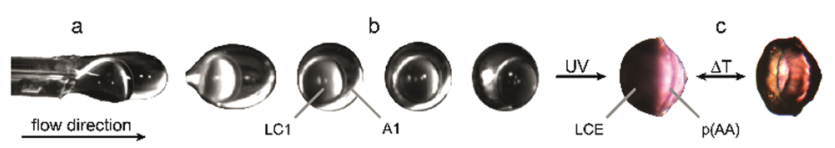

Fig. 3 (a) Formation of an acorn-like droplet at the tips of capillaries, (b) development of the droplet's morphology in the continuous flow of silicone oil and (c) shape change during the thermal phase transition of a crosslinked eccentric Janus particle after UV irradiation. acorn-like objects, especially at the boundary surface of $\mathbf{A 1}$. A large interface between $\mathbf{L C 1}$ and $\mathbf{A 1}$ is assumed to decrease the order of the mesogens at the boundary surface and thus to disturb the director field of the LC-monomer. Furthermore, the effective alignment of the mesogens and the generation of an ordered director field mainly rely on the undisturbed circulation of the fluid LC material inside the droplets, driven by the shearing of the continuously flowing PDMS. However, the non-spherical geometry of the LC1 part in the Janus droplets might have a retarding influence on the flow pattern of the LC-monomer compared to microfluidic produced almost spherical droplets of pure $\mathbf{L C 1}$. $^{42}$

Alternatively a hindrance of the shape variation of the LCE, by "gluing" it to a non-actuating polyacrylamide network, can be assumed as a possible reason. The rigid and inflexible nonactuating part of the Janus particles made from A1 is expected to decrease the shape change of the LCE during the phase transition, since both parts get chemically fixed to each other during the UV induced radical polymerization process. This limits the actuation process of the LCE at the boundary surface.

Both effects depend on the geometry of the particle and the size of the interface between both parts of the Janus particles LC1 and A1. Thus two different strategies to increase the magnitude of actuation during the phase transition were developed. The first strategy aimed at a variation of the interfacial tension between the A1-part and CP to change the particle's geometry from an acorn-like to a bicompartmental shaped Janus particle (Section 2.2). This particle morphology features a smaller, more planar boundary surface between the monomer mixtures compared to the larger, more curved boundary surface of an acorn-like particle. The second strategy aimed at the dynamic elongation of the Janus particle's geometry perpendicular to the boundary surface by increasing the shear rate acting on the droplets in the microfluidic setup. Especially the second strategy led to strongly asymmetric rod-like Janus particles (Section 2.3). Both strategies were supposed to provide a higher ratio of the LCE-volume to the size of the boundary surface in order to reduce problems in both the orientation process of the mesogens and the movability of the LCE material during the phase transition (compared to Fig. 5).

2.2 Bicompartmental Janus particles. The first approach to the formation of bicompartmental Janus particles required the decrease of the interfacial tension especially between $\mathbf{A 1}$ and the PDMS in the continuous phase CP. For this purpose two different surfactants were tested: a low molecular surfactant SDBS (sodium dodecylbenzenesulfonate) and a high molecular PDMS-PEG graft-copolymer (polydimethylsiloxanepolyethylene glycol), which is specially designed to reduce the surface tension between water and silicone oil. The influence of both surfactants on the interfacial tension between A1 and CP was studied by measuring the interfacial tension as a function of the concentration of the surfactant dissolved in the aqueous monomer mixture A1. The measurements were carried out using a Wilhelmy plate tensiometer at $85{ }^{\circ} \mathrm{C}$ which provided the same thermal conditions as the droplet formation takes place in the microfluidic reactor (see Section 1). 


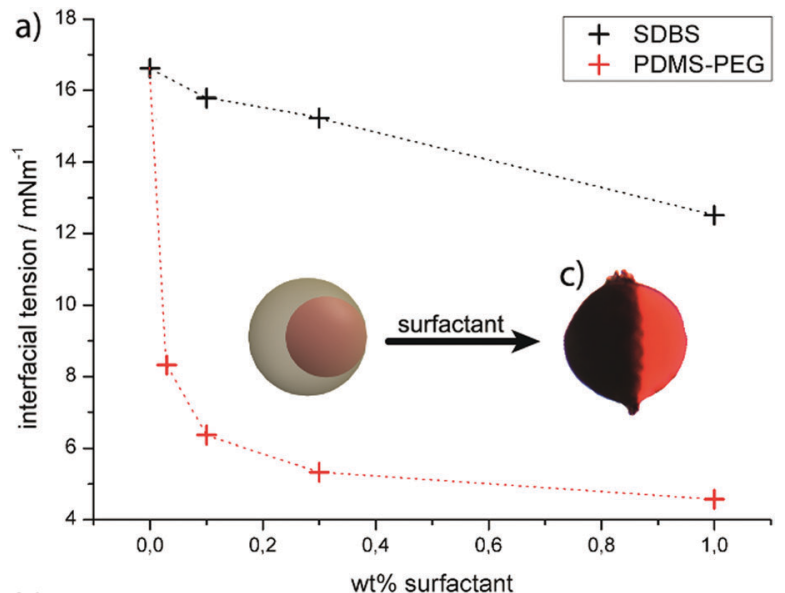

b)
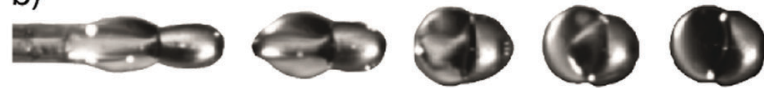

Fig. 4 (a) Interfacial tension A1/CP plotted against the concentration of the surfactants SDBS (black line) and the PDMS-PEG copolymer (red line). (b) Formation of bicompartmental Janus droplets in the microfluidic device and (c) the morphology of resulting Janus particles after UV polymerization and crosslinking.

The measurement results are illustrated in Fig. 4(a). As it was expected, both surfactants clearly induce a reduction of the interfacial tension. Thereby the PDMS-PEG copolymer exhibits a strong decrease of the interfacial tension already at very low concentrations, such as the reduction of more than $60 \%$ of its baseline value at just $0.1 \mathrm{wt} \%$. By comparison, SDBS shows a smaller influence on the interfacial tension (reduction of $8.5 \%$ at $0.1 \mathrm{wt} \%$ SDBS) and also a minor decrease at higher amounts of surfactant. This can be explained by the strong affinity of the PDMS-block for the silicone oil phase which is due to the similarities in the chemical structure and polarity.

With respect to these results, the PDMS-PEG-copolymer was used successfully as a surfactant in the aqueous phase A1, which transformed the Janus particle's morphology from an acorn-like to a bicompartimental shape. The Janus particles showed an approximately planar boundary surface, which could be observed during the droplet formation (Fig. 4(b)) and after UV-polymerization (Fig. 4(c)). The shape changes of the hemispherical shaped LCE-part were monitored by POM during the thermal phase transition, reaching relative length changes up to $15 \%$ for the new particle morphology. These results represent an improvement of the actuation properties compared to the acorn-like Janus particles introduced above. However, the actuation was still smaller than the actuating LCE particles composed of the same material. ${ }^{40-42}$ Thus to further improve the abilities of Janus particles as actuators the second strategy was carried out, too.

2.3 Rod-like elongated Janus particles. The formation of elongated rod-like Janus particles was enforced by increasing the shear rate acting on the Janus droplets inside the polymerization tube. Therefore the inner diameter of the polymerization tube was decreased from $750 \mu \mathrm{m}$ to $500 \mu \mathrm{m}$, after the droplet formation at the tips of the capillaries took place.

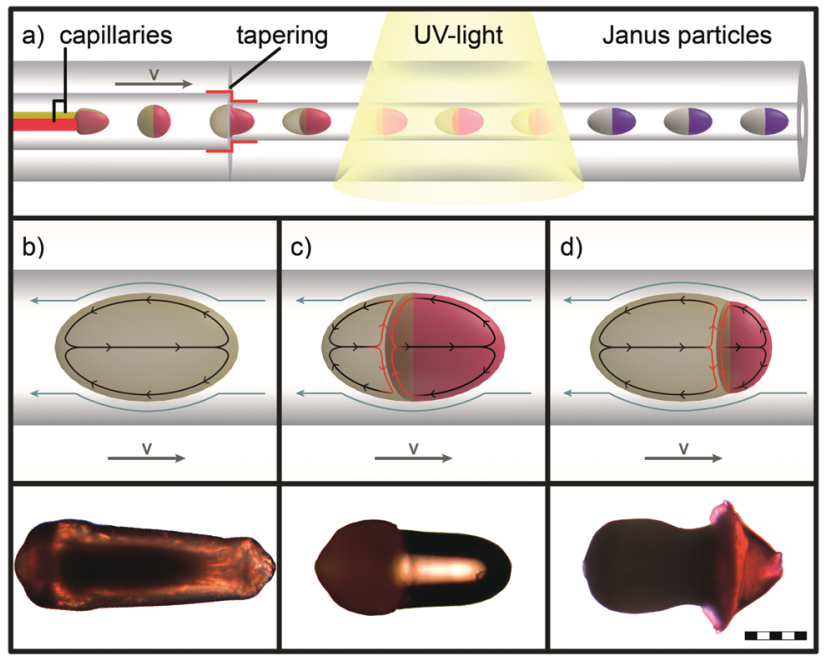

Fig. 5 (a) Formation of elongated droplets by the reduction of the polymerization tube's inner diameter. The illustrations (b)-(d) schematically illustrate three different droplet morphologies during the continuous flow as well as the streaming patterns inside the droplets (grey: LC1 and red: A1). The obtained particle morphologies after the UV polymerization and crosslinking process are shown at the bottom (scale bar: $200 \mu \mathrm{m}$ ).

The tapering of the tube's inner diameter by one third causes an increase of the flow velocity by the factor of 2.25 with respect to the continuity law and thus an increase of the shear gradient, too. This forced the spherical Janus droplets to transform into an elongated shape. ${ }^{50,51}$ Fig. 5(a) illustrates schematically the shape transformation and polymerization process.

Three different morphologies of rod-like particles were adjusted systematically by the variation of the parameters of the microfluidic setup, in order to investigate a correlation between the morphology of the particle and the magnitude of actuation, which will be discussed in Section 3.1. These different types of particles are illustrated in Fig. 5(b)-(d) and can be synthesized with a wide range of axial lengths and lateral diameters of particles as well as different ratios of the liquid crystalline elastomer LC1 to the aqueous hydrogel A1 by the variation of the flow rates of dispersed and continuous phases.

The first type of elongated particle produced by the described microfluidic method contains only the liquid crystalline monomer mixture LC1. Fig. 5(b) shows the torpedo-shaped morphology of such a rod-like LCE particle. These exhibit an axial length of 820 to $860 \mu \mathrm{m}$ and a lateral diameter up to $300 \mu \mathrm{m}$ at the back side of particles, whereas the axial diameter of the front side is reduced. The particle's morphology can be explained by means of the increased velocity gradient in the capillary, which deforms the droplets. ${ }^{50,51}$ In our case laminar flow can be expected due to low Reynold numbers, which are present under the previously described conditions in the microfluidic setup. ${ }^{41}$ This provides a parabolic profile of the flow velocity. The details of the deformation process are, however, difficult to understand, because the viscosity inside the droplets increases quickly during the UV irradiation as a result of the crosslinking polymerization, which transforms a low molar mass nematic droplet (liquid like) into a soft elastic solid. 
The UV polymerization of the droplets is completed in a short time frame (approx. 1-2 seconds), which enables the "freezing" of the elongated non-equilibrium state of the droplet morphology. The resulting torpedo-shaped, rod-like, single-phase particles enabled the determination of the LCE's maximum actuation, and were used as a benchmark for the Janus particles (see Fig. 6(d)).

The morphology of the elongated Janus particles results from an even more complex situation, as both parts of the Janus droplets do not only possess different viscosities, but also different expected viscosity increases during the polymerization, which most likely follow different kinetics. From these, elongated Janus particles of two different morphologies were achieved by the variation of the viscosity of the aqueous phase. These particles are displayed in Fig. 5(c), (d) and 6(a)-(c). The Janus particle 5.c was synthesized from the same compositions of the monomer mixtures LC1 and A1 as described previously (Sections 2.1 and 2.2). The aqueous phase of particle 5.d was replaced by the highly viscous monomer mixture A2, which contained $0.5 \mathrm{wt} \%$ of a high molecular weight poly(acrylamide) in order to strongly increase the viscosity of the aqueous phase. Particle 5.c provides a torpedo-shaped elongated morphology of the A1 part (low viscosity, colored with a red dye) and an accumulation of the LC1 part at the back side of the particle (dark colored) with respect to the flow direction. The particle features a lateral diameter of $310 \mu \mathrm{m}$. On the other side the morphology of Janus particle $5 . \mathrm{d}$ consists of a cone-shaped short A2 part (high viscosity) providing a diameter of $425 \mu \mathrm{m}$ and a strongly tapered LC1 part. The LCE again shows a lateral diameter of roughly $310 \mu \mathrm{m}$ at the back side of the particle. Due to the viscosity increase on going from A1 (Fig. 5(c)) to A2 (Fig. 5(d)) the LC1 part gets now the most strongly deformed part of the Janus particle. From the optical impression of the morphology of particle 5.d the LC1 part is supposed to be pulled apart from the aqueous A2 part during the polymerization.

The morphological difference between the particles 5.c and 5.d is reasonable, as low viscosity particles are deformed most strongly in a given shear gradient. This is appropriate for either the aqueous, non-LC phase $\mathbf{A 1}$ (particle 5.c) or the LC1-phase (particle 5.d). Furthermore, a special phenomenon of LCEs, the so-called "soft elasticity", may be important for the morphology of particle $5 . \mathrm{d}^{52-55}$ Soft elasticity is associated with the orientation of a (at first) macroscopically unoriented sample during a stretching process, as done here. It leads to a situation where the sample can be further stretched without an increase in stress. Later on, both types of particles were produced with different ratios of monomer mixtures LC1 to A1 (or A2), in order to investigate a correlation with the actuation properties. Thereby it turned out that the variability of the volume ratios was larger for Janus particles from LC1 and A2 than for LC1 and A1 combinations, because Janus particles with a high LC1 ratio broke up too easily in combination with the low viscosity $\mathbf{A 1}$ phase at the tips of the capillaries.

\section{Characterization of the rod-like Janus particles}

3.1 Actuation properties. The investigation of the actuation of the elongated rod-like Janus particles was carried out by observing the length changes of the LCE part parallel to the particle's long axis at the thermal phase transition. To follow the shape change accurately the particles were tempered on a hot stage, using a silicone oil matrix to guarantee a uniform heating over the whole particle, and further analyzed by polarized optical microscopy. The LCE's phase transition occurred in the temperature range of 80 to $130{ }^{\circ} \mathrm{C}$, featuring a length contraction along the particle's long axis and an expansion of the particle's diameter (see Fig. 6 and Table 1). This leads to the conclusion that the mesogens are oriented in a bipolar director field, which would lead to an expansion perpendicular to the director and a subsequent contraction along the director during the phase transition. Further WAXS studies proved the assumed bipolar director field of the mesogen (Section 3.2). The entire actuation process is very fast (about 1 second) and completely reversible during both the heating and the cooling process. This process can be watched in a real-time video of an actuating Janus particle (see Video S2, $\mathrm{ESI} \dagger$ ), which is placed on a hot stage at $130{ }^{\circ} \mathrm{C}$ and cooled down from the isotropic to the glassy state by cold air streams very fast.

The influence of different synthetic and microfluidic parameters on the actuation properties of particles was studied. Fig. 6 illustrates the actuation of differently synthesized rod-like particles and highlights the length changes of the particle's LCE part during the phase transition. The influence on the actuation properties was studied by the variation of (1) the viscosities of aqueous monomer mixtures $\mathbf{A 1}$ and $\mathbf{A 2}$ (particle 6.a and b), (2) the volume ratio of the polymerized parts $\mathbf{L C} \mathbf{1}$ to $\mathbf{A 2}$ (particle 6.b and c)

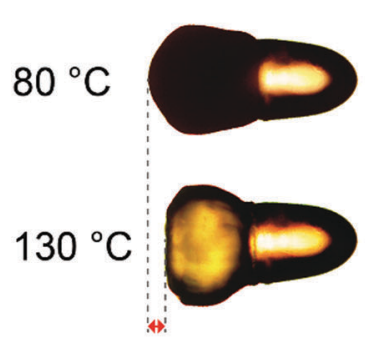

a

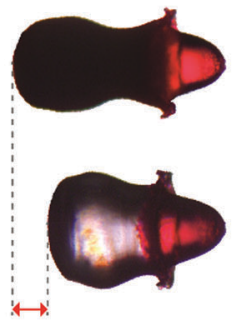

b

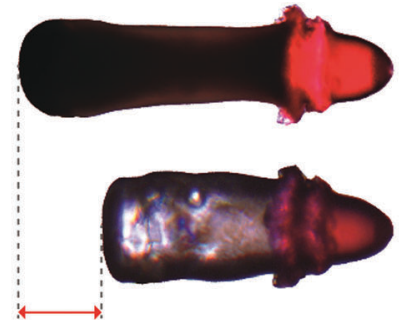

C

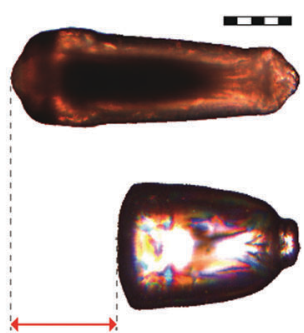

d

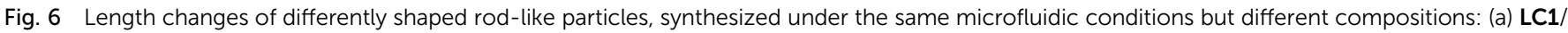

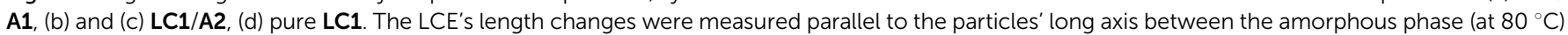
and the isotropic phase (at $130{ }^{\circ} \mathrm{C}$ ). The calculated relative length changes are (a) $21 \%$, (b) $40 \%$, (c) $52 \%$, and (d) $58 \%$ (scale bar: $200 \mu \mathrm{m}$ ). 
and (3) the presence of the aqueous polymer $\mathbf{A} 2$ and thus the presence of a boundary surface with the LCE (particle 6.c and d). All particles were synthesized under the same microfluidic conditions in terms of the temperature, UV irradiation, the polymerization tube's inner diameter and the continuous phase' viscosity and flow rate.

The different Janus particle morphologies, which were adjusted by the variation of the viscosities of the aqueous phases (Section 2.3), show a strong impact on the actuation of the LCE. The relative length changes of particle $6 . \mathrm{b}(40 \%$, high viscous mixture A2) reach almost twice the value of particle 6.a (21\%, low viscous mixture A1). Especially the centrally tapered part of the LCE in particle 6.b features a strong actuation, which is observable by the video of the corresponding Janus particle actuation (S2, ESI $\dagger$ ). The huge difference in the actuation properties between particles $6 . \mathrm{a}$ and 6.b is probably based on the different amount of uniformly aligned mesogens along the director. In Janus droplets with the highly viscous monomer mixture A2, the LC1 part is stretched most strongly during the UV-polymerization (Section 2.3). Deformation is thereby facilitated by the soft elasticity of the LCE. This stretching improves the orientation of the LC director and creates a bipolar orientation field. This explains the improved actuation properties of particle 6.b as a result of an increased viscosity of the aqueous monomer mixture.

However, the spherically shaped back side of the particle's LCE exhibits a weak actuation during the phase transition for all the differently synthesized particles. This is the result of the bipolar orientation field of the mesogens, which demands the bending of the director towards the defect point located at the outermost back side of the LCE part (see Fig. 8(e)). Since the volume of the poorly actuating back side of the LCE is roughly constant over the different particles (at similar particle diameters), the actuation of a rod-like Janus particle should improve by increasing the total volume of the LCE. This assumption was proved by increasing the ratio of monomer mixture LC1 to A2 from 2:1 (particle 6.b) up to $4: 1$ (particle 6.c), which further improved the relative length changes up to $52 \%$.

The third analyzed parameter was the effect of the boundary surface between the LCE and the aqueous polymer $\left(\mathbf{B}_{\mathbf{L C} / \mathbf{A}}\right)$, the impact of which on the LCE's actuation properties was studied. As mentioned before (Section 2.1), the interface between LC1 and the non-actuating $\mathbf{A 1}$ or $\mathbf{A} 2$ is supposed to be problematic for the actuation process. Since (1) the flow alignment in the area of the boundary surface is probably less effective and (2) the direct contact between both phases hinders the deformation of the LCE. Both effects would be decreased in the absence of the boundary surface $\mathbf{B}_{\mathbf{L C} / \mathbf{A}}$. Thus Janus particle 6.c was synthesized with the same volume of LCE and under the same microfluidic conditions as used for the synthesis of the single phase LCE particle 6.d. Upon comparing the actuation properties, it is found that particle 6.d features an improved relative length change of $58 \%$ in the absence of the boundary surface $\mathbf{B}_{\mathbf{L C} / \mathbf{A}}$, featuring the benchmark for the actuation process of the rod-like particles.

Both negative effects, the presence of the boundary surface and the bending director at the LCE part's back side, are

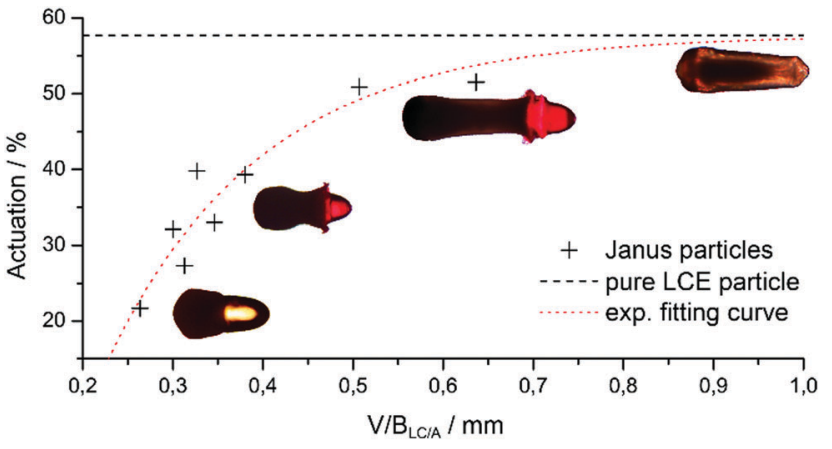

Fig. 7 Actuation of differently shaped and composited Janus particles as a function of $\mathbf{V} / \mathbf{B}_{\mathbf{L C} / \mathbf{A}}$, which reflects the LCE volume divided by the area of $\mathbf{B}_{\mathrm{LC} / \mathbf{A}}$. By increasing the value of $\mathbf{V} / \mathbf{B}_{\mathrm{LC} / \mathbf{A}}$, the approximate linear slope at small values flattens at higher values and converges at a constant saturation value of $58 \%$, reflecting the maximum actuation measured for the single phase LCE particle.

supposed to become negligible by increasing the volume of the LCE part while keeping the area of $\mathbf{B}_{\mathbf{L C} / \mathbf{A}}$ as low as possible. Thus the actuation of differently synthesized Janus particles was correlated with the calculated quantity $\mathbf{V} / \mathbf{B}_{\mathbf{L C} / \mathbf{A}}$, which correlates with the LCE volume divided by the area of the boundary surface LC/A. The corresponding data are illustrated in Fig. 7. An exponential fit function was selected to fit the data, which converge for high values of $\mathbf{V} / \mathbf{B}_{\mathbf{L C} / \mathbf{A}}$ to the actuation benchmark of the single phase LCE particle 6.d. These data clearly illustrate that the disturbing effects on the mesogen's alignment at the boundary surface and the defect point can be minimized by an optimization of $\mathbf{V} / \mathbf{B}_{\mathbf{L C} / \mathbf{A}}$ and the microfluidic parameters. This establishes a systematic microfluidic synthesis of actuating Janus particles, featuring different morphologies, various sizes and high actuation values that reach almost the length changes of single phase LCE particles.

3.2 WAXS measurements. Wide angle X-ray scattering (WAXS) experiments were performed on an elongated rod-like Janus particle to study the mesogen's alignment and to determine the direction of the LCE's director field. The alignment of the LCE's director dictates the actuation properties of the particles, which are discussed in Section 2.2. Different regions of a Janus particle were measured by using a narrow X-ray beam of roughly $100 \mu \mathrm{m}$ diameter. Fig. 8 illustrates the results of the X-ray measurements, carried out at three different measuring points along the particle's $z$-axis (Fig. 8(b)). The measurement positions were chosen (1) at the actuating center of the particle's LCE, (2) at the boundary surface of the LCE and the aqueous polymer network and (3) within the aqueous part $\mathbf{A 1}$ of the particle.

The scattering pattern of the measurement within the LCE at position 1 is illustrated in Fig. 8(a). The corresponding scattering intensity profile along the scattering angle $2 \theta$ is plotted in Fig. 8(d) featuring two scattering maxima, which are typically expected for nematic liquid crystals. ${ }^{43}$ The maximum at small angles reflects the mean distance of the mesogens in the direction of the director (first peak in pair correlation function) and was calculated to be $18.0 \AA$ using the Bragg equation. 

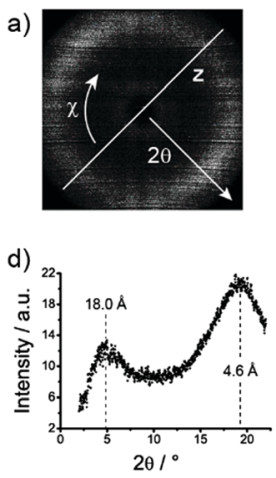

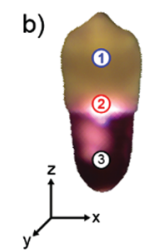

e)

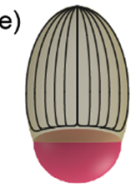

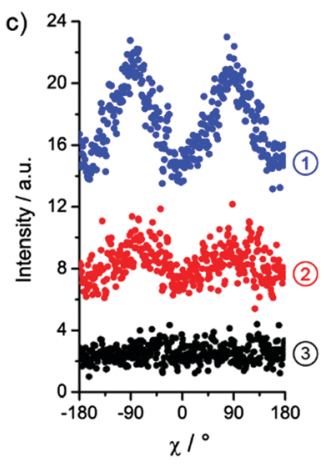

Fig. 8 Wide angle $X$-ray scattering of an elongated rod-like Janus particle. (a) X-ray scattering pattern of the measurement within the LCE (position 1). It shows two intensity maxima perpendicular to the particle's $z$-axis. (b) Different measurement positions on the analyzed Janus particle. (c) Radial integrated intensities over the wide angle arc $\chi$, which depicts two intensity maxima at $-90^{\circ}$ and $90^{\circ}$ within the LCE. (d) Scattering intensity depending on the scattering angle $2 \theta$, which exhibits two maxima at $4.9^{\circ}$ and $19.2^{\circ}$. (e) Schematic representation of the mesogen's bipolar director field within the LCE part of the Janus particles.

The second maximum at wide angles corresponds to the mean lateral distance of the mesogens, which was calculated to be $4.6 \AA$.

The determination of the director's orientation was performed by analysis of the azimuthal intensity distribution $I(\chi)$. This diffused wide and small angle peaks were radially integrated over $\chi$ from $2 \theta=3^{\circ}$ to $6^{\circ}$ and $2 \theta=15^{\circ}$ to $22^{\circ}$, respectively. The results are displayed in Fig. $8(\mathrm{c})$. The measurements at position 1 exhibit two intensity maxima at wide-angle arcs of approximately $\chi=-90^{\circ}$ and $\chi=90^{\circ}$, which indicates the director's alignment along the $z$-axis of the particle and proves the assumption of the mesogen's alignment in a predicted bipolar director field (Fig. 8(e)) along the particle's long axis (Section 2.3). The examination of the results at position 2 further verifies a decrease of the mesogens' alignment along the director field, since the maxima appear clearly less pronounced. At position 3 both maxima disappear according to the expected isotropic arrangement of the aqueous polymer network.

\section{Conclusions}

For the first time, actuating liquid crystalline Janus particles can be synthesized in a microfluidic co-flow device by photo-polymerizing both parts of the liquid Janus droplets simultaneously. Thereby a hydrophobic, shape changing LCE "half-droplet" gets glued to a hydrophilic, non-actuating "half-droplet", made of a crosslinked polymer hydrogel. To obtain the maximum shape change of the LCE at the phase transition temperature, the Janus droplet's morphology is modified by systematically varying the microfluidic parameters. The minimization of the interface between both dispersed phases of the Janus droplets turns out to be the crucial factor to increase the actuating properties of the LCE. Besides adjusting the interfacial tensions of the dispersed monomer phases with respect to the silicone-oil used as the continuous phase in the microreactor, the deformation of the Janus droplets into an elongated rod-like structure by increasing the shear rate in the polymerization tube especially leads to Janus particles with strong shape changes of the LCE during the phase transition. The resulting Janus particles feature highly reproducible and completely reversible actuating properties of the LCE, which are as strong as those of monophasic LCE particles processed under the same conditions. Additionally, the amphiphilic character of the presented Janus particles opens possibilities for self-organization and a further implementation of other functional materials besides the LCE as the hydrophilic part of Janus particles.

\section{Acknowledgements}

T. Hessberger and L. B. Braun acknowledge funding by the German Science Foundation (DFG: Ze 230/24-1). F. Henrich acknowledges funding by the ERC, Advanced grant SUPRO 340391 (HJB).

\section{References}

1 P. G. de Gennes, Science, 1992, 256, 495-497.

2 A. Walther and A. H. E. Müller, J. Am. Chem. Soc., 2013, 113, 5194-5261.

3 J. Du and R. K. O'Reilly, Chem. Soc. Rev., 2011, 40, 2402-2416.

4 J. Hu, S. Zhou, Y. Sun, X. Fang and L. Wu, Chem. Soc. Rev., 2012, 41, 4356.

5 C. Kaewsaneha, P. Tangboriboonrat, D. Polpanich, M. Eissa and A. Elaissari, Colloids Surf., A, 2013, 439, 35-42.

6 X. Pang, C. Wan, M. Wang and Z. Lin, Angew. Chem., Int. Ed., 2014, 53, 5524-5538.

7 D. Baah and T. Floyd-Smith, Microfluid. Nanofluid., 2014, 17, 431-455.

8 S. Lone and I. W. Cheong, RSC Adv., 2014, 4, 13322.

9 X. T. Sun, M. Liu and Z. R. Xu, Talanta, 2014, 121, 163-177.

10 C. A. Serra, I. U. Khan, Z. Chang, M. Bouquey, R. Muller, I. Kraus, M. Schmutz, T. Vandamme, N. Anton, C. Ohm, R. Zentel, A. Knauer and M. Köhler, J. Flow Chem., 2013, 3, 66-75.

11 S. Bhaskar, J. Hitt, S. W. L. Chang and J. Lahann, Angew. Chem., Int. Ed., 2009, 48, 4589-4593.

12 S. Y. Lee and S. Yang, Chem. Commun., 2015, 51, 1639-1642. 13 A. Synytska, R. Khanum, L. Ionov, C. Cherif and C. Bellmann, ACS Appl. Mater. Interfaces, 2011, 3, 1216-1220. 14 S. Berger, L. Ionov and A. Synytska, Adv. Funct. Mater., 2011, 21, 2338-2344.

15 J. W. Kim, J. Cho, J. Cho, B. J. Park, Y.-J. Kim, K.-H. Choi and J. W. Kim, Angew. Chem., 2016, 15588, 4509-4513.

16 F. Liang, K. Shen, X. Qu, C. Zhang, Q. Wang, J. Li, J. Liu and Z. Yang, Angew. Chem., Int. Ed., 2011, 50, 2379-2382.

17 A. Walther, M. Hoffmann and A. H. E. Müller, Angew. Chem., Int. Ed., 2008, 47, 711-714. 
18 T. Nisisako, T. Torii, T. Takahashi and Y. Takizawa, Adv. Mater., 2006, 18, 1152-1156.

19 C. H. Chen, A. R. Abate, D. Lee, E. M. Terentjev and D. A. Weitz, Adv. Mater., 2009, 21, 3201-3204.

20 J. Ge, Y. Hu, T. Zhang and Y. Yin, J. Am. Chem. Soc., 2007, 129, 8974-8975.

21 S. Berger, A. Synytska, L. Ionov, K. Eichhorn and M. Stamm, Macromolecules, 2008, 41, 9669-9676.

22 A. Synytska and L. Ionov, Part. Part. Syst. Charact., 2013, 30, 922-930.

23 M. a. C. Stuart, W. T. S. Huck, J. Genzer, M. Müller, C. Ober, M. Stamm, G. B. Sukhorukov, I. Szleifer, V. V. Tsukruk, M. Urban, F. Winnik, S. Zauscher, I. Luzinov and S. Minko, Nat. Mater., 2010, 9, 101-113.

24 U. Choudhury, L. Soler, J. G. Gibbs, S. Sanchez and P. Fischer, Chem. Commun., 2015, 51, 8660-8663.

25 W. Gao, M. D’Agostino, V. Garcia-Gradilla, J. Orozco and J. Wang, Small, 2013, 9, 467-471.

26 H. R. Jiang, N. Yoshinaga and M. Sano, Phys. Rev. Lett., 2010, 105, 1-4.

27 M. Amouzadeh Tabrizi and M. Shamsipur, RSC Adv., 2015, 5, 51508-51511.

28 P. G. de Gennes, C. R. Hebd. Seances Acad. Sci., Ser. B, 1975, 281, 101.

29 R. Zentel, Angew. Chem., Int. Ed. Engl., 1989, 28, 1407-1415. 30 H. Finkelmann, Makromol. Chem., 1981, 2, 317-322.

31 P. Xie and R. Zhang, J. Mater. Chem., 2005, 15, 2529.

32 G. N. Mol, K. D. Harris, C. W. M. Bastiaansen and D. J. Broer, Adv. Funct. Mater., 2005, 15, 1155-1159.

33 P. Beyer, E. M. Terentjev and R. Zentel, Macromol. Rapid Commun., 2007, 28, 1485-1490.

34 C. Ohm, N. Haberkorn, P. Theato and R. Zentel, Small, 2011, 7, 194-198.

35 E. K. Fleischmann and R. Zentel, Angew. Chem., Int. Ed., 2013, 52, 8810-8827.

36 T. H. Ware and T. J. White, Polym. Chem., 2015, 6, 4835-4844. 37 C. L. Van Oosten, D. Corbett, D. Davies, M. Warner, C. W. M. Bastiaansen and D. J. Broer, Macromolecules, 2008, 41, 8592-8596.

38 S. Schuhladen, F. Preller, R. Rix, S. Petsch, R. Zentel and H. Zappe, Adv. Mater., 2014, 26, 7247-7251.
39 C. Ohm, M. Brehmer and R. Zentel, Adv. Mater., 2010, 22, 3366-3387.

40 C. Ohm, C. Serra and R. Zentel, Adv. Mater., 2009, 21, 4859-4862.

41 C. Ohm, E. K. Fleischmann, I. Kraus, C. Serra and R. Zentel, Adv. Funct. Mater., 2010, 20, 4314-4322.

42 C. Ohm, N. Kapernaum, D. Nonnenmacher, F. Giesselmann, C. Serra and R. Zentel, J. Am. Chem. Soc., 2011, 133, 5305-5311.

43 E.-K. Fleischmann, H.-L. Liang, N. Kapernaum, F. Giesselmann, J. Lagerwall and R. Zentel, Nat. Commun., 2012, 3, 1178.

44 I. U. Khan, C. A. Serra, N. Anton, X. Li, R. Akasov, N. Messaddeq, I. Kraus and T. F. Vandamme, Int. J. Pharm., 2014, 473, 239-249.

45 Y. T. Yang, J. Wei, X. Li, L. J. Wu, Z. Q. Chang and C. A. Serra, Adv. Powder Technol., 2015, 26, 156-162.

46 D. L. Thomsen, P. Keller, J. Naciri, R. Pink, H. Jeon, D. Shenoy and B. R. Ratna, Macromolecules, 2001, 34, 5868-5875.

47 T. J. Suen, Y. Jen and J. V. Lockwood, J. Polym. Sci., 1958, 31, 481-497.

48 L. B. Braun, T. Hessberger, C. A. Serra and R. Zentel, Macromol. React. Eng., 2016, DOI: 10.1002/mren.201600015.

49 X. Ge, J. Huang, J. Xu, J. Chen and G. Luo, Soft Matter, 2016, 12, 3425-3430.

50 A. Gupta and M. Sbragaglia, Phys. Rev. E: Stat., Nonlinear, Soft Matter Phys., 2014, 90, 1-17.

51 P. Tanpaiboonkul, W. Lerdwijitjarud, A. Sirivat and R. G. Larson, Polymer, 2007, 48, 3822-3835.

52 S. M. Clarke, A. R. Tajbakhsh, E. M. Terentjev, C. Remillat, G. R. Tomlinson and J. R. House, J. Appl. Phys., 2001, 89, 6530-6535.

53 H. Search, C. Journals, A. Contact, M. Iopscience and I. P. Address, J. Phys.: Condens. Matter, 1999, 11, 239-257.

54 P. Martinoty, P. Stein, H. Finkelmann, H. Pleiner and H. R. Brand, Eur. Phys. J. E: Soft Matter Biol. Phys., 2004, 14, 311-321.

55 H. R. Brand, H. Pleiner and P. Martinoty, Soft Matter, 2006, 2, 182. 